\title{
Understanding language change
}

Proc. Natl Acad. Sci. USA 114, E8822-E8829 (2017)

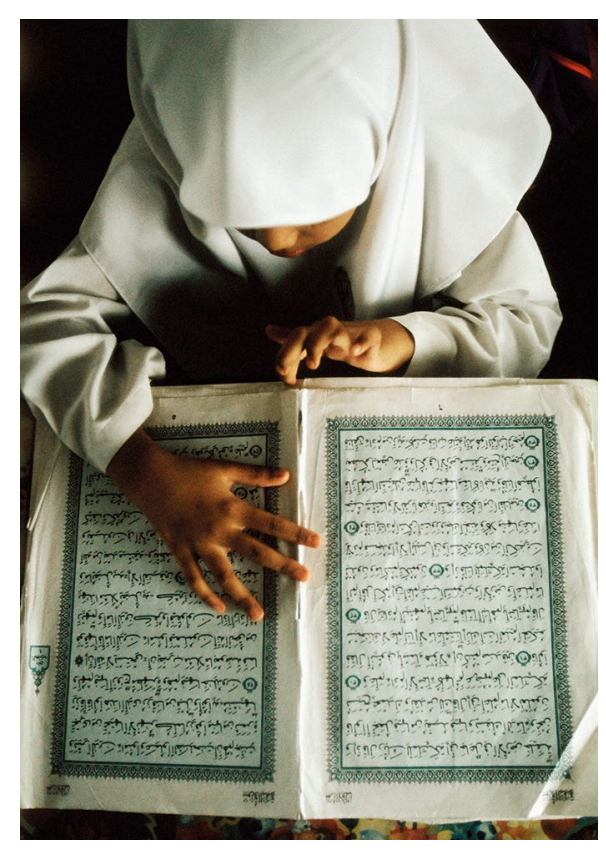

Credit: ASIA IMAGES GROUP PTE LTD / ALAMY STOCK PHOTO

Languages change over time, which makes it harder to trace their history and genealogy further back than 10,000 years. However, it is unclear whether all aspects of language, for instance, grammar and vocabulary, change at the same rate. If not, identifying rates of change for different linguistic features may enable us to reconstruct language history more accurately.

In new research, Simon Greenhill and colleagues ask whether different aspects of language evolve in different ways and at different rates. The authors apply Bayesian phylogenetic modelling to grammatical structures and basic vocabulary items from 81 Austronesian languages to infer rates of change. Unlike previous research, they find that grammatical structures changed much more rapidly over time than basic vocabulary. The grammatical features the authors studied were distributed more or less evenly in three rate-of-change categories: fast, medium and slow. Basic vocabulary items, however, nearly all fell into the slow category. The grammatical structures that changed slowly appeared to be consistent with earlier reports and tended to be more abstract than faster-changing grammatical features.

This work suggests that looking at language change in a more fine-grained manner may offer more reliable information on which to base inferences about language history and genealogy, extending further into the past.

\section{Stavroula Kousta}

Published online: 2 November 2017 https://doi.org/10.1038/s41562-017-0250-y 